University of New Mexico

UNM Digital Repository

Mathematics and Statistics Faculty and Staff

Publications

Academic Department Resources

2017

\title{
A Bipolar Single Valued Neutrosophic Isolated Graphs: Revisited
}

Florentin Smarandache

Said Broumi

Assia Bakali

Mohamed Talea

Mohsin Khan

Follow this and additional works at: https://digitalrepository.unm.edu/math_fsp

Part of the Logic and Foundations Commons, Number Theory Commons, and the Other Mathematics Commons 


\title{
A Bipolar Single Valued Neutrosophic Isolated Graphs: Revisited
}

Said Broumi

Laboratory of Information Processing, Faculty of Science Ben M'Sik, University Hassan II, B.P 7955,Sidi Othman, Casablanca, Morocco broumisaid78@gmail.com

Mohamed Talea

Laboratory of Information Processing, Faculty of Science Ben M'Sik, University Hassan II, B.P 7955,Sidi Othman, Casablanca, Morocco taleamohamed@yahoo.fr

\author{
Assia Bakali \\ Ecole Royale Navale ,Boulevard Sour Jdid,B.P \\ 16303 Casablanca, Morocco \\ assiabakali@yahoo.fr
}

Florentin Smarandache

Department of Mathematics, University of New Mexico, 705 Gurley Avenue, Gallup, NM, 87301, USA

fsmarandache@gmail.com; smarand@unm.edu

\author{
Mohsin Khan \\ Department of Mathematics, Abdul Wali Khan University, \\ Mardan 23200, Pakistan \\ mohsinkhan7284@gmail.com
}

\begin{abstract}
In this research paper, the graph of the bipolar single-valued neutrosophic set model (BSVNS) is proposed. The graphs of single valued neutrosophic set models is generalized by this graph. For the BSVNS model, several results have been proved on complete and isolated graphs. Adding, an important and suitable condition for the graphs of the BSVNS model to become an isolated graph of the BSVNS model has been demonstrated.
\end{abstract}

\section{KEYWORDS}

Bipolar single valued neutrosophic graphs (BSVNG), complete-BSVNG, isolated-BSVNGs.

\section{Introduction}

The concept of 'Neutrosophic logic' was developed by Prof. Dr. F. Smarandache in 1995 and get published in 1998. "It is a branch of philosophy which studies the origin, nature, and scope of neutralities, as well as their interactions with different ideational spectra"[4]. The concepts of fuzzy sets [8] and intuitionistic fuzzy set [6] were generalized by adding an independent indeterminacy-membership. Neutrosophic logic is a powerful tool to deal with incomplete, indeterminate, and inconsistent information, which is the main reason for widespread concerns of researchers. The concept of neutrosophic set(NS for short) is characterized by three independent degrees namely truthmembership degree (T), indeterminacymembership degree (I), and falsity-membership degree (F).To practice NSs in real life situations efficiently, The subclass of the neutrosophic sets called single-valued neutrosophic set (in short SVNS) was defined by Smarandache in [4]. In another paper [5], Wang et al. defined the various operations and operators for the SVNS model. In [11] Deli et al. proposed a new concept called bipolar neutrosophic sets. This concept appear as a generalization of fuzzy sets, intuitionistic fuzzy sets, bipolar fuzzy sets, bipolar intuitionistic fuzzy sets and single valued neutrosophic set. The benefits of applying the NSs have been addressed in [18].The theory of graphs is the mostly used tool for resolving combinatorial problems in diverse disciplines like computer science, algebra and topology, etc. In $[2,4]$ Smarandache proposed two kinds of neutrosophic graphs to deal with situations in which there exist inconsistencies and indeterminacies among the vertices which cannot be dealt with by fuzzy graphs and different hybrid structures including bipolar fuzzy graphs, intuitionistic fuzzy graphs, bipolar intuitionsitc 
fuzzy graphs $[1,7,9,10]$, The first kind is based on literal indeterminacy (I) component, the second kind of neutrosophic graphs is based on numerical truth-values (T, I, F), Recently, a hybrid study by combining SVNS and classical graph theory was carried out and that concept is called Single valued neutrosophic graph (SVNG) was presented by Broumi et al [12, 13, 14, 17, $20,22]$.In addition, the concept of bipolar neutrosophic set was combined with graph theory and new graph model was presented. This concept is called bipolar single valued neutrosophic graph (BSVNGs). In $[15,16]$ Broumi et al. proposed the concept of bipolar single valued neutrosophic graph as a generalized the concept of fuzzy graph, intuitionistic fuzzy graph, bipolar fuzzy graph and single valued neutrosophic graph.

The objective of this article is to demonstrate the essential and satisfactory condition of BSVNGs to be an isolated-BSVNG.

\section{Background of research}

Some of the important background knowledge in this paper is presented in this section. These results can be found in $[4,5,12,13,15,21]$.

Definition 2.1 [4] Le $\zeta$ be a universal set. The neutrosophic set $A$ on the universal set $\zeta$ categorized into three membership functions called the true membership function $T_{A}(\mathrm{x})$, indeterminate membership function $I_{A}(\mathrm{x})$ and false membership function $F_{A}(\mathrm{x})$ contained in real standard or non-standard subset of $]^{-} 0,1^{+}[$ respectively and satisfy the following condition

${ }^{-} 0 \leq \sup \mathrm{T}_{\mathrm{A}}(\mathrm{x})+\sup \mathrm{I}_{\mathrm{A}}(\mathrm{x})+\operatorname{supF}_{\mathrm{A}}(\mathrm{x}) \leq 3^{+}$ (1)

Definition 2.2 [5] Let $\zeta$ be a universal set. The single valued neutrosophic sets (SVNs) A on the universal $\zeta$ is denoted as following

$$
\mathrm{A}=\left\{<x: \mathrm{T}_{\mathrm{A}}(\mathrm{x}), \mathrm{I}_{\mathrm{A}}(\mathrm{x}), \mathrm{F}_{\mathrm{A}}(\mathrm{x})>\mathrm{x} \in \zeta\right\}
$$

The functions $\mathrm{T}_{\mathrm{A}}(\mathrm{x}) \in[0.1], \mathrm{I}_{\mathbf{A}}(\mathrm{x}) \in[0.1]$ and $\mathrm{F}_{\mathrm{A}}(\mathrm{x}) \in[0.1]$ are called " degree of truth, indeterminacy and falsity membership of $\mathrm{x}$ in A", satisfy the following condition:

$$
0 \leq T_{A}(\mathrm{x})+I_{A}(\mathrm{x})+F_{A}(\mathrm{x}) \leq 3
$$

Definition 2.3 [12] A SVNG of $G^{*}=(\mathrm{V}, \mathrm{E})$ is a graph $\mathrm{G}=(\mathrm{A}, \mathrm{B})$ where

a. The following memberships: $T_{A}: \mathrm{V} \rightarrow[0,1]$, $I_{A}: V \rightarrow[0,1]$ and $F_{A}: V \rightarrow[0,1]$ represent the truth, indeterminate and false membership degrees of $x \in V$ respectively and

$$
\begin{aligned}
& 0 \leq T_{A}(w)+I_{A}(w)+F_{A}(w) \leq 3 \\
& \forall w \in V
\end{aligned}
$$

b. The following memberships: $T_{B}: \mathrm{E} \rightarrow[0,1]$, $I_{B}: \mathrm{E} \rightarrow[0,1]$ and $\mathrm{F}_{\mathrm{B}}: \mathrm{E} \rightarrow[0,1]$ are defined by

$\mathrm{T}_{\mathrm{B}}(v, w) \leq \min \left[\mathrm{T}_{\mathrm{A}}(v), \mathrm{T}_{\mathrm{A}}(w)\right]$

$I_{B}(v, w) \geq \max \left[\mathrm{I}_{\mathrm{A}}(v), \mathrm{I}_{\mathrm{A}}(w)\right]$ and

$F_{B}(v, w) \geq \max \left[\mathrm{F}_{\mathrm{A}}(v), \mathrm{F}_{\mathrm{A}}(w)\right]$

Represent the true, indeterminate and false membership degrees of the $\operatorname{arc}(v, w) \in(\mathrm{V} \times \mathrm{V})$, where

$$
\begin{aligned}
& 0 \leq \mathrm{T}_{\mathrm{B}}(v, w)+\mathrm{I}_{\mathrm{B}}(v, w)+\mathrm{F}_{\mathrm{B}}(v, w) \leq 3 \\
& \forall(v, w) \in \mathrm{E}
\end{aligned}
$$

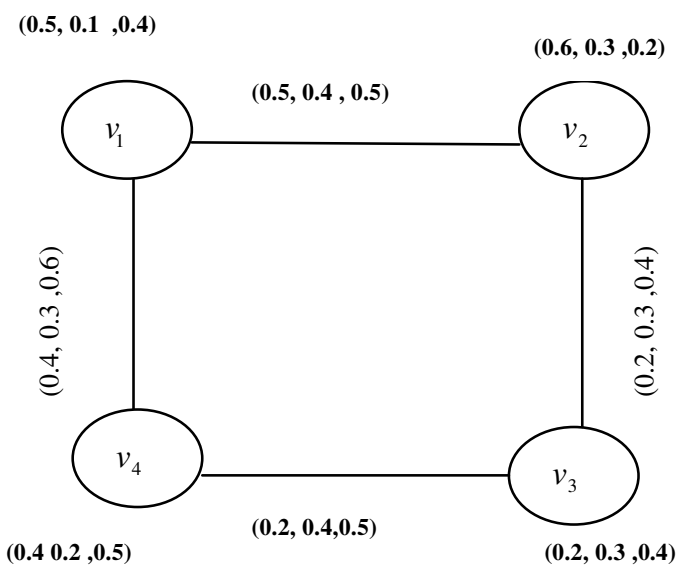

Fig.1.SVN-graph

Definition 2.4 [12]. A SVNG $\mathrm{G}=(\mathrm{A}, \mathrm{B})$ is named a complete-SVNG if

$$
\begin{aligned}
& \mathrm{T}_{\mathrm{B}}(v, w)=\min \left[\mathrm{T}_{\mathrm{A}}(\mathrm{v}), \mathrm{T}_{\mathrm{A}}(w)\right] \\
& \mathrm{I}_{\mathrm{B}}(v, w)=\max \left[\mathrm{I}_{\mathrm{A}}(\mathrm{v}), \mathrm{I}_{\mathrm{A}}(w)\right] \\
& \mathrm{F}_{\mathrm{B}}(v, w)=\max \left[\mathrm{F}_{\mathrm{A}}(\mathrm{v}), \mathrm{F}_{\mathrm{A}}(w)\right]
\end{aligned}
$$


$\forall v, w \in \mathrm{V}$

Definition 2.5[12]. Let $\mathrm{G}=(\mathrm{A}, \mathrm{B})$ be SVNG. Hence, the complement of SVNG G on $G^{*}$ is a SVNG $\bar{G}$ on $G^{*}$ where

a. $\bar{A}=\mathrm{A}$

b. $\bar{T}_{A}(w)=T_{A}(w), \overline{I_{A}}(w)=I_{A}(w), \overline{F_{A}}(w)=F_{A}(w)$

$\forall w \in V$

c. $\overline{T_{\mathrm{B}}}(v, w)=\min \left[\mathrm{T}_{\mathrm{A}}(v), \mathrm{T}_{\mathrm{A}}(w)\right]-\mathrm{T}_{\mathrm{B}}(v, w)$

$\overline{\mathrm{I}_{\mathrm{B}}}(v, w)=\max \left[I_{A}(v), I_{A}(w)\right]-I_{B}(v, w)(15)$

$\overline{\mathrm{F}_{\mathrm{B}}}(v, w)=\max \left[F_{A}(v), F_{A}(w)\right]-F_{B}(v, w)$, $\forall(v, w) \in \mathrm{E}$.

Definition 2.6 [15].A BSVNG $G=\left(\right.$ A, B)of $G^{*}$ $=(\mathrm{V}, \mathrm{E})$ is a partner such that $\mathrm{A}=\left(\mathrm{T}_{\mathrm{A}}^{\mathrm{P}}, \mathrm{I}_{\mathrm{A}}^{\mathrm{P}}, \mathrm{F}_{\mathrm{A}}^{\mathrm{P}}, \mathrm{T}_{\mathrm{A}}^{\mathrm{N}}\right.$ $\left., \mathrm{I}_{\mathrm{A}}^{\mathrm{N}}, \mathrm{F}_{\mathrm{A}}^{\mathrm{N}}\right)$ is a $\mathrm{BSVNS}$ in $\mathrm{V}$ and $\mathrm{B}=\left(\mathrm{T}_{\mathrm{B}}^{\mathrm{P}}, \mathrm{I}_{\mathrm{B}}^{\mathrm{P}}, \mathrm{F}_{\mathrm{B}}^{\mathrm{P}}, \mathrm{T}_{\mathrm{B}}^{\mathrm{N}}\right.$ $\left., \mathrm{I}_{\mathrm{B}}^{N}, \mathrm{~F}_{\mathrm{B}}^{\mathrm{N}}\right)$ is a BSVNS in $\mathrm{E}$ such that

(i) $\mathrm{T}_{\mathrm{B}}^{\mathrm{P}}(v, w) \leq \min \left(\mathrm{T}_{\mathrm{A}}^{\mathrm{P}}(v), \mathrm{T}_{\mathrm{A}}^{\mathrm{P}}(w)\right)$ and $\mathrm{T}_{\mathrm{B}}^{\mathrm{N}}(v, w) \geq \max \left(\mathrm{T}_{\mathrm{A}}^{\mathrm{N}}(v), \mathrm{T}_{\mathrm{A}}^{\mathrm{N}}(w)\right)$ (17)

(ii) $\mathrm{I}_{\mathrm{B}}^{\mathrm{P}}(v, w) \geq \max \left(\mathrm{I}_{\mathrm{A}}^{\mathrm{P}}(v), \mathrm{I}_{\mathrm{A}}^{\mathrm{P}}(w)\right) \quad$ and $\mathrm{I}_{\mathrm{B}}^{\mathrm{N}}(v, w) \leq \min \left(\mathrm{I}_{\mathrm{A}}^{\mathrm{N}}(\mathrm{v}), \mathrm{I}_{\mathrm{A}}^{\mathrm{N}}(w)\right)$ (18)

(iii) $\mathrm{F}_{\mathbf{B}}^{\mathrm{P}}(v, w) \geq \max \left(\mathrm{F}_{\mathrm{A}}^{\mathrm{P}}(v), \mathrm{F}_{\mathbf{A}}^{\mathrm{P}}(w)\right)$ and $\mathrm{F}_{\mathrm{B}}^{\mathrm{N}}(v, w) \leq \min \left(\mathrm{F}_{\mathrm{A}}^{\mathrm{N}}(v), \mathrm{F}_{\mathrm{A}}^{\mathrm{N}}(w)\right)$, $\forall(\mathrm{v}, \mathrm{w}) \in \mathrm{E}$

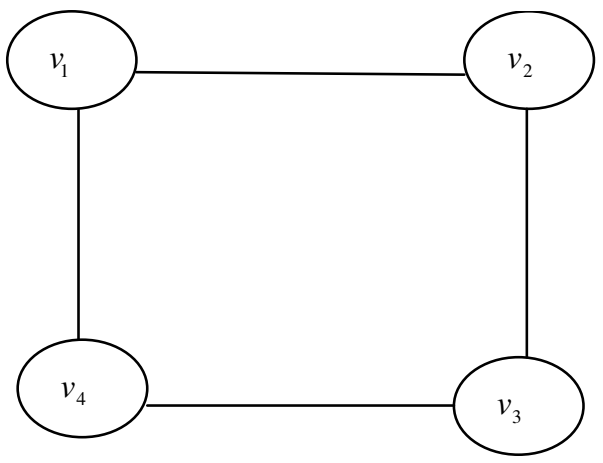

Fig.2 BSVNG

\begin{tabular}{|l|c|}
\hline$v_{1}$ & The values of vertex \\
\hline$v_{1}$ & $(0.2,0.2,0.4,-0.4,-0.1,-0.4)$ \\
\hline$v_{2}$ & $(0.1,0.3,0.5,-0.6,-0.2,-0.3)$ \\
\hline$v_{3}$ & $(0.2,0.3,0.5,-0.3,-0.2,-0.1)$ \\
\hline$v_{4}$ & $(0.3,0.2,0.4,-0.2,-0.3,-0.5)$ \\
\hline
\end{tabular}

Table1. The values of vertex of BSVNG

\begin{tabular}{|c|c|}
\hline & The values of edge \\
\hline$v_{12}$ & $(0.1,0.3,0.6,-0.2,-0.3,-0.1)$ \\
\hline$v_{23}$ & $(0.1,0.3,0.6,-0.1,-0.6,-0.7)$ \\
\hline$v_{34}$ & $(0.1,0.5,0.6,-0.1,-0.6,-0.5)$ \\
\hline$v_{14}$ & $(0.2,0.3,0.5,-0.2,-0.3,-0.5)$ \\
\hline
\end{tabular}

Definition 2.7 [15]. The complement of BSVNG $\mathbf{G}=(A, B)$ of $G^{*}=(\mathrm{V}, \mathrm{E})$ is a BSVNG $\bar{G}=(\bar{A}$, $\bar{B})$ of $G^{*}=(\mathrm{V}, \mathrm{E})$ such that

(i) $\bar{A}=\mathrm{A}=\left(\mathrm{T}_{\mathrm{A}}^{\mathrm{P}}, \mathrm{I}_{\mathrm{A}}^{\mathrm{P}}, \mathrm{F}_{\mathrm{A}}^{\mathrm{P}}, \mathrm{T}_{\mathrm{A}}^{\mathrm{N}}, \mathrm{I}_{\mathrm{A}}^{\mathrm{N}}, \mathrm{F}_{\mathrm{A}}^{\mathrm{N}}\right)$ and

(ii) $\overline{\mathrm{B}}=\left(\mathrm{T}_{\overline{\mathrm{B}}}^{\mathrm{P}}, \mathrm{I}_{\overline{\mathrm{R}}}^{\mathrm{P}}, \mathrm{F}_{\overline{\mathrm{R}}}^{\mathrm{P}}, \mathrm{T}_{\overline{\mathrm{B}}}^{\mathrm{N}}, \mathrm{I}_{\overline{\mathrm{R}}}^{\mathrm{N}}, \mathrm{F}_{\overline{\mathrm{R}}}^{\mathrm{N}}\right)$ on $\mathrm{E}=\mathrm{V} \times \mathrm{V}$ is defined as

$\mathrm{T}_{\overline{\mathrm{B}}}^{\mathrm{P}}(v, w)=\min \left(\mathrm{T}_{\mathrm{A}}^{\mathrm{P}}(v), \mathrm{T}_{\mathrm{A}}^{\mathrm{P}}(w)\right)-\mathrm{T}_{\mathrm{B}}^{\mathrm{P}}(v, w)$

$\mathrm{T}_{\overline{\mathrm{R}}}^{\mathrm{N}}(v, w)=\max \left(\mathrm{T}_{\mathrm{A}}^{\mathrm{N}}(v), \mathrm{T}_{\mathrm{A}}^{\mathrm{N}}(w)\right)-\mathrm{T}_{\mathrm{B}}^{\mathrm{N}}(v, w)(20)$

$\mathrm{I}_{\overline{\mathrm{B}}}^{\mathrm{P}}(\mathrm{v}, \mathrm{w})=\max \left(\mathrm{I}_{\mathrm{A}}^{\mathrm{P}}(v), \mathrm{I}_{\mathrm{A}}^{\mathrm{P}}(w)\right) \mathrm{I}_{\mathrm{B}}^{\mathrm{P}}(\mathrm{v}, \mathrm{w})$

$\mathrm{I}_{\overline{\mathrm{R}}}^{\mathrm{N}}(\mathrm{v}, \mathrm{w})=\min \left(\mathrm{I}_{\mathrm{A}}^{\mathrm{N}}(v), \mathrm{I}_{\mathrm{A}}^{\mathrm{N}}(w)\right) \mathrm{I}_{\mathrm{B}}^{\mathrm{N}}(\mathrm{v}, \mathrm{w})$

$\mathrm{F}_{\overline{\mathrm{B}}}^{\mathrm{P}}(v, w)=\max \left(\mathrm{F}_{\mathrm{A}}^{\mathrm{P}}(v), \mathrm{F}_{\mathrm{A}}^{\mathrm{P}}(w)\right)-\mathrm{F}_{\mathrm{B}}^{\mathrm{P}}(v, w)$

$\mathrm{F}_{\overline{\mathbf{R}}}^{\mathrm{N}}(v, w) \min \left(\mathrm{F}_{\mathrm{A}}^{\mathrm{N}}(v),(w)\right)-\mathrm{F}_{\mathbf{B}}^{\mathrm{N}}(v, w)$,

$\forall(v, \mathrm{w}) \in \mathrm{E}$

Definition 2.8 [15]. A BSVNG $G=(A, B)$ is called a complete-BSVNG if

$\mathrm{T}_{\mathrm{B}}^{\mathrm{P}}(v, w)=\min \left(\mathrm{T}_{\mathrm{A}}^{\mathrm{P}}(v), \mathrm{T}_{\mathrm{A}}^{\mathrm{P}}(w)\right), \quad(23)$

$\mathrm{T}_{\mathrm{B}}^{\mathrm{N}}(v, w)=\max \left(\mathrm{T}_{\mathrm{A}}^{\mathrm{N}}(v), \mathrm{T}_{\mathrm{A}}^{\mathrm{N}}(w)\right), \quad(24)$

$\mathrm{I}_{\mathrm{B}}^{\mathrm{P}}(v, w)=\max \left(\mathrm{I}_{\mathrm{A}}^{\mathrm{P}}(v), \mathrm{I}_{\mathrm{A}}^{\mathrm{P}}(w)\right)$,

$\mathrm{I}_{\mathrm{B}}^{\mathrm{N}}(v, w)=\min \left(\mathrm{I}_{\mathrm{A}}^{\mathrm{N}}(v), \mathrm{I}_{\mathrm{A}}^{\mathrm{N}}(w)\right)$

$\mathrm{F}_{\mathbf{B}}^{\mathrm{P}}(v, w)=\max \left(\mathrm{F}_{\mathrm{A}}^{\mathrm{P}}(v), \mathrm{F}_{\mathrm{A}}^{\mathrm{P}}(w)\right)$,

$\mathrm{F}_{\mathbf{B}}^{\mathrm{N}}(v, w)=\min \left(\mathrm{F}_{\mathrm{A}}^{\mathrm{N}}(\mathrm{v}), \mathrm{F}_{\mathrm{A}}^{\mathrm{N}}(w)\right)$

(28) $\forall v, w \in \mathrm{V}$

Definition 2.9[7]. The complement of BIFG $\mathbf{G}=$ $(A, B)$ of $G^{*}=(\mathrm{A}, \mathrm{B})$ is a BIFG $\bar{G}=(\bar{A}, \bar{B})$ of $\overline{G^{*}}=(\mathrm{V}, \mathrm{V} x \mathrm{~V})$ where $\bar{A}=\mathrm{A}=\left(\mathrm{T}_{\mathrm{A}}^{\mathrm{P}}, \mathrm{F}_{\mathrm{A}}^{\mathrm{P}}, \mathrm{T}_{\mathrm{A}}^{\mathrm{N}}, \mathrm{F}_{\mathrm{A}}^{\mathrm{N}}\right)$ and $\overline{\mathrm{B}}=\left(\overline{\mathrm{T}_{\mathrm{B}}^{\mathrm{p}}}, \overline{\mathrm{F}_{\mathrm{B}}^{\mathrm{P}}}, \overline{\mathrm{T}_{\mathrm{B}}^{\mathrm{N}}}, \overline{\mathrm{F}_{\mathrm{B}}^{\mathrm{N}}}\right)$ are defined as

$\overline{\mathrm{T}}_{\mathrm{B}}^{\mathrm{P}}(v, w)=\min \left(\mathrm{T}_{\mathrm{A}}^{\mathrm{P}}(v), \mathrm{T}_{\mathrm{A}}^{\mathrm{P}}(w)\right)-\mathrm{T}_{\mathrm{B}}^{\mathrm{P}}(v, w)(29)$

$\overline{\mathrm{F}}_{\mathbf{B}}^{\mathrm{P}}(v, w)=\max \left(\mathrm{F}_{\mathrm{A}}^{\mathrm{P}}(v), \mathrm{F}_{\mathrm{A}}^{\mathrm{P}}(w)\right)-\mathrm{F}_{\mathbf{B}}^{\mathrm{P}}(v, w)(30)$

$\mathrm{T}_{\mathrm{B}}^{\mathrm{N}}(v, w)=\max \left(\mathrm{T}_{\mathrm{A}}^{\mathrm{N}}(v), \mathrm{T}_{\mathrm{A}}^{\mathrm{N}}(w)\right)-\mathrm{T}_{\mathrm{B}}^{\mathrm{N}}(v, w)(31)$ $\overline{\mathbf{F}}_{\mathbf{B}}^{\mathbf{N}}(v, w) \min \left(\mathrm{F}_{\mathbf{A}}^{\mathrm{N}}(v),(w)\right)-\mathbf{F}_{\mathbf{B}}^{\mathbf{N}}(v, w) \quad \forall v$, $\mathrm{w} \in \mathrm{V}, v w \in \widetilde{\mathrm{V}}^{2}(32)$

Theorem 2.10[13]Let $\mathrm{G}=(\mathrm{A}, \mathrm{B})$ be a SVNG, then the SVNG is called an isolated-SVNG if 
and only if the complement of $\mathrm{G}$ is a completeSVNG.

Theorem 2.11[21]Let $G=(A, B)$ be a $F G$, then the FG is called an isolated-FG if and only if the complement of $\mathrm{G}$ is a complete- FG

\section{MAin RESUlts}

Theorem 3.1: A BSVNG $=(\mathrm{A}, \mathrm{B})$ is an isolatedBSVNG iff the complement of BSVNG is a complete- BSVNG.

Proof: Let $G=(A, B)$ be a complete- BSVNG.

Therefore $\mathrm{T}_{\mathrm{B}}^{\mathrm{P}}(v, w)=\min \left(\mathrm{T}_{\mathrm{A}}^{\mathrm{P}}(\mathrm{v}), \mathrm{T}_{\mathrm{A}}^{\mathrm{P}}(\mathrm{w})\right)$,

$\mathrm{T}_{\mathrm{B}}^{\mathrm{n}}(v, w)=\max \left(\mathrm{T}_{\mathrm{A}}^{\mathrm{n}}(v), \mathrm{T}_{\mathrm{A}}^{\mathrm{n}}(w)\right)$,

$\mathrm{I}_{\mathrm{B}}^{\mathrm{P}}(v, w)=\max \left(\mathrm{I}_{\mathrm{A}}^{\mathrm{P}}(v), \mathrm{I}_{\mathrm{A}}^{\mathrm{P}}(w)\right)$,

$\mathrm{I}_{\mathrm{B}}^{\mathrm{n}}(v, w)=\min \left(\mathrm{I}_{\mathrm{A}}^{\mathrm{n}}(v), \mathrm{I}_{\mathrm{A}}^{\mathrm{n}}(w)\right)$,

$\mathrm{F}_{\mathbf{B}}^{\mathrm{P}}(v, w)=\max \left(\mathrm{F}_{\mathbf{A}}^{\mathrm{P}}(v), \mathrm{F}_{\mathbf{A}}^{\mathrm{P}}(w)\right)$,

$\mathrm{F}_{\mathrm{B}}^{\mathrm{n}}(v, w)=\min \left(\mathrm{F}_{\mathrm{A}}^{\mathrm{n}}(\mathrm{v}), \mathrm{F}_{\mathrm{A}}^{\mathrm{n}}(w)\right), \forall v, w \in \mathrm{V}$.

Hence in $\overline{\mathrm{G}}$,

$\bar{T}_{B}^{P}(v, w)=\min \left(T_{A}^{P}(v), T_{A}^{P}(w)\right)-T_{B}^{P}(v, w)$

$=\min \left(T_{A}^{P}(v), T_{A}^{P}(w)\right)-\min \left(T_{A}^{P}(v), T_{A}^{P}(w)\right)$

$=0$

and

$\bar{I}_{B}^{P}(\mathrm{v}, \mathrm{w})=\max \left(I_{A}^{P}(\mathrm{v}), I_{A}^{p}(\mathrm{w})\right)-I_{B}^{P}(\mathrm{v}, \mathrm{w})$

$=\max \left(I_{A}^{P}(\mathrm{v}), I_{A}^{P}(\mathrm{w})\right)-\max \left(I_{A}^{P}(\mathrm{v}), I_{A}^{P}(\mathrm{w})\right)$

$=0$

In addition

$\bar{F}_{B}^{p}(v, w)=\max \left(F_{A}^{P}(v), F_{A}^{p}(w)\right)-F_{B}^{P}(v, w)$

$=\max \left(F_{A}^{P}(v), F_{A}^{P}(w)\right)-\max \left(F_{A}^{P}(v), F_{A}^{P}(w)\right)$

$=0$

We have for the negative membership edges

$\bar{T}_{B}^{N}(v, w)=\max \left(\mathrm{T}_{\mathrm{A}}^{\mathrm{N}}(v), \mathrm{T}_{\mathrm{A}}^{\mathrm{N}}(w)\right)-T_{B}^{N}(v, w)$

$=\max \left(T_{A}^{N}(v), T_{A}^{N}(w)\right)-\max \left(\mathrm{T}_{\mathrm{A}}^{\mathrm{N}}(v), \mathrm{T}_{\mathrm{A}}^{\mathrm{N}}(w)\right)$
$=0$ and

$\bar{I}_{B}^{N}(v, w)=\min \left(I_{A}^{N}(v), I_{A}^{N}(w)\right)-I_{B}^{N}(v, w)$

$=\min \left(I_{A}^{N}(v), I_{A}^{N}(w)\right)-\min \left(I_{A}^{N}(\mathrm{v}), I_{A}^{N}(\mathrm{w})\right)$

$=0$

In addition

$\bar{F}_{B}^{N}(v, w)=\min \left(F_{A}^{N}(v), F_{A}^{N}(w)\right)-F_{B}^{N}(v, w)$

$=\min \left(F_{A}^{N}(v), F_{A}^{N}(w)\right)-\min \left(F_{A}^{N}(v), F_{A}^{N}(w)\right)$

$=0$

So $\left(\bar{T}_{B}^{P}(v, w), \overline{\mathrm{I}}_{\mathrm{B}}^{\mathrm{p}}(v, w), \bar{F}_{B}^{P}(v, w), \bar{T}_{B}^{N}(v, w)\right.$, $\left.\bar{I}_{B}^{N}(v, w), \bar{F}_{B}^{N}(v, w)\right)=(0,0,0,0,0,0)$

Hence $\mathrm{G}=(A, B)$ is an isolated-BSVNGs

Proposition 3.2: The notion of isolatedBSVNGs generalized the notion of isolated fuzzy graphs.

Proof: If the value of $\mathrm{I}_{\mathrm{A}}^{\mathrm{P}}(w)=\mathrm{F}_{\mathrm{A}}^{\mathrm{P}}(w)=\mathrm{T}_{\mathrm{A}}^{\mathrm{n}}(w)=$ $\mathrm{I}_{\mathrm{A}}^{\mathrm{n}}(w)=\mathrm{F}_{\mathrm{A}}^{\mathrm{n}}(w)=0$, then the notion of isolatedBSVNGs is reduced to isolated fuzzy graphs.

Proposition 3.3: The notion of isolatedBSVNGs generalized the notion of isolatedSVNGs.

Proof: If the value of $\mathrm{T}_{\mathrm{A}}^{\mathrm{n}}(w)=\mathrm{I}_{\mathrm{A}}^{\mathrm{n}}(w)=\mathrm{F}_{\mathrm{A}}^{\mathrm{n}}(w)=$ 0 , then the concept of isolated-BSVNGs is reduced to isolated-SVNGs.

Proposition 3.4: The notion of isolatedBSVNGs generalized the notion of isolatedbipolar intuitionistic fuzzy graph.

Proof: If the value of $\mathrm{I}_{\mathrm{A}}^{\mathrm{p}}(w)=\mathrm{I}_{\mathrm{A}}^{\mathrm{n}}(w)$, then the concept of isolated-BSVNGs is reduced to isolated-bipolar intutuitionistic fuzzy graphs

\section{IV.COMPARTIVE STUDY}

In this section, we present a table showing that the bipolar single valued neutrosophic graph generalized the concept of the crisp graph, fuzzy graph [9], intuitionistic fuzzy graph[1], bipolar fuzzy graph[10], bipolar intuitionistic fuzzy graph[7] and single valued neutrosophic graph[12].

For convenience we denote

F-graph : Fuzzy graphs 
IF-graph: Intuitionistic fuzzy graph

BF-graph: Bipolar fuzzy graph

BIF-graph: Bipolar intutionistic fuzzy graph

SVN-graph: Single valued neutrosophic graph BSVN-graph: Bipolar single valued neutrosophic graph

\begin{tabular}{|c|c|c|c|c|c|c|}
\hline \multirow{2}{*}{$\begin{array}{l}\text { Type } \\
\text { of } \\
\text { graphs }\end{array}$} & \multicolumn{6}{|c|}{ The membership values of vertex/ edge } \\
\hline & $\mathrm{T}_{\mathrm{A}}^{\mathrm{p}}(w)$ & $\mathrm{I}_{\mathrm{A}}^{\mathrm{p}}(w)$ & $\mathrm{F}_{\mathrm{A}}^{\mathrm{p}}(w)$ & $\mathrm{T}_{\mathrm{A}}^{\mathrm{n}}(w)$ & $\mathrm{I}_{\mathrm{A}}^{\mathrm{n}}(w)$ & $\mathrm{F}_{\mathrm{A}}^{\mathrm{n}}(w)$ \\
\hline $\begin{array}{l}\text { crisp } \\
\text { graph }\end{array}$ & 1 or 0 & 0 & 0 & 0 & 0 & 0 \\
\hline$\overline{F G}$ & $\begin{array}{l}\in \\
{[0,1]}\end{array}$ & 0 & 0 & 0 & 0 & 0 \\
\hline IFG & $\begin{array}{l}\in \\
{[0,1]}\end{array}$ & 0 & $\begin{array}{l}\in \\
{[0,1]}\end{array}$ & 0 & 0 & 0 \\
\hline SVNG & $\begin{array}{l}\in \\
{[0,1]}\end{array}$ & $\begin{array}{l}\in \\
{[0,1]}\end{array}$ & $\begin{array}{l}\in \\
{[0,1]}\end{array}$ & 0 & 0 & 0 \\
\hline BFG & $\begin{array}{l}\in \\
{[0,1]}\end{array}$ & 0 & 0 & $\begin{array}{l}\in \\
{[-1,0]}\end{array}$ & 0 & 0 \\
\hline BIFG & $\begin{array}{l}\in \\
{[0,1]}\end{array}$ & 0 & $\begin{array}{l}\in \\
{[0,1]}\end{array}$ & $\begin{array}{l}\in \\
{[-1,0]}\end{array}$ & 0 & $\begin{array}{l}\in \\
{[-1,0]}\end{array}$ \\
\hline $\begin{array}{l}\text { BSVN } \\
\text { G }\end{array}$ & $\begin{array}{l}\in \\
{[0,1]}\end{array}$ & $\begin{array}{l}\in \\
{[0,1]}\end{array}$ & $\begin{array}{l}\in \\
{[0,1]}\end{array}$ & $\begin{array}{l}\in \\
{[-1,0]}\end{array}$ & $\begin{array}{l}\in \\
{[-1,0]}\end{array}$ & $\begin{array}{l}\in \\
{[-1,0]}\end{array}$ \\
\hline
\end{tabular}

Table3. Different types of graphs

Neutrosophic graph is the generalization of crisp graph, fuzzy graph, intuitionistic fuzzy graph, bipolar fuzzy graph, bi-polar intuitionistic fuzzy graph and single-valued neutrosophic graph. In this table, we can see that by removing the indeterminacy and non-membership values from neutrosophic graph, the neutrosophic graph reduces to fuzzy graph. By removing the indeterminacy value from neutrosophic graph, the neutrosophic graph reduces to intuitionistic fuzzy graph. Similarly, by removing the positive and negative indeterminacy and nonmembership values from bi-polar neutrosophic graph, the bi-polar neutrosophic graph reduces to bi-polar fuzzy graph. By removing the positive and negative indeterminacy values from bi-polar neutrosophic graph, the bi-polar neutrosophic graph reduces to bi-polar intuitionistic fuzzy graph. By the similar way, we can reduce a bipolar single valued neutrosophic graph to a neutrosophic graph by removing the negative membership, indeterminacy and nonmembership values.

\section{CONCLuSion}

In this article, we have proved necessary and sufficient condition under which BSVNGs is an isolated-BSVNGs. The notion of isolated-
BSVNGs generalized the isolated-fuzzy graph and isolated- SVNGs. In addition, in future research, we shall concentrate on extending the idea of this paper by using the interval valued bipolar neutrosophic graph as a generalized form of bipolar neutrosophic graph.

\section{REFERENCES}

1. Gani, A. and Shajitha, B.S: Degree: Order and size in Intuitionistic Fuzzy Graphs. International Journal of Algorithms, Computing and Mathematics, 3(3) (2010).

2. Smarandache, F.: Refined Literal Indeterminacy and the Multiplication Law of Sub -Indeterminacies. Neutrosophic Sets and Systems, Vol.9, 58-63 (2015).

3. Smarandache, F.: Symbolic Neutrosophic Theory, Europanova asbl, Brussels, (2015)

4. Smarandache, F.: Neutrosophy, Neutrosophic Probability, Sets and Logic, Proquest Information \& Learning, Ann Arbor, Michigan, USA, 105p,1998

5. Wang, H., Smarandache, F., Zhang, Y., and Sunderraman, R.: Single Valued Neutrosophic Sets. Multispace and Multistructure 4, 410-413 (2010).

6. Atanassov,K. : Intuitionistic Fuzzy Sets. Fuzzy Sets and Systems, Vol.20, 87-96 (1986).

7. Sankar, K., Ezhilmaran, D.: Balanced bipolar intuitionistic fuzzy graphs, International Research Journal of Engineering and Technology (IRJET), Volume: 03 Issue: 11, 806-812 (2016).

8. Zadeh, L.: Fuzzy sets. Information and Control, 8 338-35 (1965).

9. Bhattacharya, P.: Some Remarks on Fuzzy Graphs. Pattern Recognition Letters 6, 297-302 (1987).

10. Akram, M.: Bipolar Fuzzy Graphs. Information Science, doi:10.1016/j.ins.2011.07.037, (2011).

11. Deli, I., Ali, M., Smarandache, :Bipolar Neutrosophic Sets and Their Application Based on Multi-criteria Decision Making Problems, in: Advanced Mechatronic Systems (ICAMechS) 249- 254 (2015).

12. Broumi, S., Talea, M., Bakali, Smarandache, F.: Single Valued Neutrosophic Graphs. Journal of New Theory, N 10, 86-101(2016).

13. Broumi, S., Bakali, A., Talea, M., Smarandache, F.: Isolated Single Valued Neutrosophic Graphs. Neutrosophic Sets and Systems, Vol.11, 74-78 (2016).

14. Broumi, S., Talea, M., Smarandache ,F. and Bakali, A.: Single Valued Neutrosophic Graphs: Degree, Order and Size. IEEE International Conference on Fuzzy Systems, 2444-2451(2016).

15. Broumi, S., Smarandache, F., Talea, M. and Bakali, A.: An Introduction to Bipolar Single Valued Neutrosophic Graph Theory. Applied Mechanics and Materials, vol .841, 184- 191(2016).

16. Broumi, S., Talea, M., Bakali, A., Smarandache, F.: On Bipolar Single Valued Neutrosphic Graphs. Journal of New Theory, N11, 84-102 (2016). 
17. Broumi, S., Bakali, A., Talea, M., Hassan, A., Smarandache, F.: Generalized Single Valued Neutrosophic Graphs of First Type,2017 IEEE International Conference on INnovations in Intelligent SysTems and Applications (INISTA), Gdynia Maritime University, Gdynia, Poland, 3-5 July ,413420 (2017).

18. http://fs.gallup.unm.edu /NSS.

19. Hassan, A., Malik, M. A., Broumi, S., Bakali, A., Talea, M. and Smarandache, F.: Special Types of Bipolar Single Valued Neutrosophic Graphs, Annals of Fuzzy Mathematics and Informatics, Volume 14, No. 1, 55-73 (2017).

20. Broumi, S., Bakali, A., Talea, M., Smarandache, F.: Generalized Bipolar Neutrosophic Graphs of Type 1, 20th International Conference on Information Fusion Xi'an, China - July 10-13, 1714-1720 (2017).

21. Rahurikar, S. : On Isolated Fuzzy Graph, International Journal of Research in Engineering Technology and Management, Vol2,Issue 06, 1-3 (2014).

22. Broumi, S., Dey, A., Bakali, A., Talea, M., Smarandache, F., Son, L. H. and Koley D.: Uniform Single Valued Neutrosophic Graphs, Neutrosophic Sets and Systems, Vol. 17, 42-49 (2017)

23. Safar, M., Mahdi, F., and Mahdi, K., An Algorithm for Detecting Cycles in Undirected Graphs using CUDA Technology, International Journal on New Computer Architectures and Their Applications (IJNCAA) 2(1): 193-212 (2012) 